\title{
Impact of Lymph Node Yield on Outcome of Patients with Head and Neck Cancer and pNO Neck
}

\author{
SASKIA MERZ ${ }^{1}$, NINA TIMMESFELD ${ }^{2}$, BORIS A. STUCK ${ }^{1}$ and SUSANNE WIEGAND ${ }^{3}$ \\ ${ }^{1}$ Department of Otolaryngology, Head and Neck Surgery, \\ University Hospital Marburg, Philipps-Universität Marburg, Marburg, Germany; \\ ${ }^{2}$ Institute of Medical Biometrics and Epidemiology, Phillips-University Marburg, Marburg, Germany; \\ ${ }^{3}$ Department of Otolaryngology, Head and Neck Surgery, University Hospital Leipzig, Leipzig, Germany
}

\begin{abstract}
Background/Aim: The extent of neck dissection in patients with head and neck cancer (HNSCC) and NO neck remains controversial. The aim of this study was to evaluate the influence of lymph node yield on overall survival in patients with pathologically node-negative HNSCC. Patients and Methods: A retrospective analysis was performed on 157 pNO HNSCC patients undergoing primary surgery with neck dissection between 2000 and 2014. The patients were divided into three groups according to the lymph node count using the optimal cut-points method. The Kaplan-Meier method and logrank test were used to analyze overall survival and compare survival curves. Results: The lymph node count cut-offs were determined to be 15 and 40. A total of 22 patients had 1-14, 79 patients had $15-39$ and 56 patients had 40 or more harvested lymph nodes. There were significant differences in survival between all groups $(p=0.001)$. Conclusion: A lymph node yield of at least 15 nodes was significantly associated with better overall survival in pathologically node-negative patients who underwent surgical resection of HNSCC.
\end{abstract}

The presence of lymph node metastasis is an important prognostic factor in head and neck squamous cell carcinoma (HNSCC). The extent of neck dissection in HNSCC with clinical N0 (cN0) neck has been a topic for debate. It is widely accepted that neck dissection is the best course for HNSCC if the possibility of neck metastasis is greater than $15-20 \%$. A prospective, randomized study on this topic showed that elective neck dissection resulted in higher survival rates than therapeutic neck dissection among patients with early-stage oral squamous cell carcinoma (1). It is still disputable whether

Correspondence to: Prof. Dr. Susanne Wiegand, Department of Otolaryngology, Head and Neck Surgery, University Hospital Leipzig, Liebigstrasse 12, 04109 Leipzig, Germany. Tel: +49 3419721720, Fax: +49 34197133, e-mail: Susanne.wiegand@ medizin.uni-leipzig.de

Key Words: Lymph node, neck dissection, nodal yield, HNSCC. a better prognosis can be achieved by increasing the number of dissected lymph nodes. A more extensive lymphadenectomy may remove a greater burden of microscopic metastatic disease and improve diagnostic accuracy by redistributing patients with otherwise unrecognized nodal disease. Improved prognosis following more extensive lymphadenectomy has been demonstrated in colon, breast, gastric, esophageal and bladder cancer (2-6). Some studies deemed that extensive neck dissection increases survival in HNSCC. Ebrahimi et al. (7) showed that nodal yield is an independent prognostic factor in patients undergoing elective neck dissection for $\mathrm{cN} 0$ oral $\mathrm{SCC}$ regardless of post-operative nodal status. They recommended that an adequate lymphadenectomy in this setting should include at least 18 nodes. Although $27 \%$ of patients in this study proved to have a pathological $\mathrm{N}+(\mathrm{pN}+)$ neck, increased lymph node yield was also demonstrated to confer survival benefit in the $\mathrm{pN} 0$ patient population. This was confirmed by Lemieux et al. (8) in pN0 patients with SCC of the oral cavity. However, whether the better prognosis can be achieved by increasing the number of dissected neck lymph nodes in patients with HNSCC of different localizations is still disputable. The objective of this study was to investigate the prognostic value of retrieved lymph nodes count in patients with node-negative HNSCC.

\section{Patients and Methods}

The retrospective study described herein consisted of 157 consecutive patients who underwent curative resection for histologically confirmed HNSCC between January 2000 and December 2014. In all patients a unilateral or bilateral neck dissection was performed depending on tumor localization and extent and they were staged as pNO. Patients with distant metastasis at diagnosis were excluded. All neck dissections were performed in a standardized manner by experienced head and neck surgeons. Operation specimens were routinely fixed in formalin, embedded in paraffin and stained with hematoxylin-eosin and then examined by a pathologist. The patient charts were retrospectively examined for demographic and pathological data including age at diagnosis, sex, tumor localization, tumor stage, grading, number of harvested 
lymph nodes, receipt of adjuvant radio(chemo)therapy and recurrence/distant metastasis. Survival was calculated from the time of diagnosis to the time of death. Baseline characteristics were described as a number (\%) and median.

Statistical analysis. The optimal cut-points method for survival data as defined by Contal et al. (9) and Mandrekar et al. (10) (R package survMisc) were applied in order to detect the greatest actuarial survival difference among the resulting subgroups. This method determined the cut-point yielding the most significant prognostic differences between two groups. Differences among the subgroups were detected based on the magnitude of the log-rank statistic test (11).

The Kaplan-Meier method was used to analyze overall survival rates according to these subgroups, and log-rank test was used to compare survival between obtained subgroups.

\section{Results}

A total of 157 patients, including 120 (76.4\%) men and 37 (23.6\%) women, were eligible for this analysis. The primary sites were the larynx in 56 patients $(35.7 \%)$, the oropharynx in 48 patients $(30.6 \%)$, the oral cavity in 43 patients $(27.4 \%)$, and the hypopharynx in 10 patients $(6.4 \%)$. Most of the patients had T1- (42.7\%) and T2-tumors (38.2\%). All patients were treated by surgery. A unilateral neck dissection was performed in 92 patients, a bilateral neck dissection in 75 patients. At least one lymph node was examined in all patients. The first cutpoint for lymph node count was 15 , dividing the patient collective into two subgroups with a significant difference in the survival probability among both groups $(p=0.0004)$ (Figure 1 ). The second group was subdivided by a second cut-point at 40 harvested lymph nodes, leading to three groups (Figure 2). The results showed that there were significant differences in the survival probability among all groups, including 22 patients with 1-14 harvested lymph nodes (group 1), 79 patients with 15-39 harvested lymph nodes (group 2) and 56 patients with 40 or more harvested lymph nodes (group 3) $(p=0.0011)$. Patient characteristics according to the number of retrieved lymph nodes are shown in Table I. Median lymph node yield was 10.5 in group 1, 26 in group 2 and 53 in group 3 (Table II). All of these lymph nodes were pathologically negative for malignancy. Overall, $22.9 \%$ of the patients had adjuvant radio(chemo)therapy (Table II). The median follow-up was 34.7 months for patients who had $\leq 14$ lymph nodes retrieved, 52.5 months for patients who had 15-39 lymph nodes retrieved and 58.2 months for patients who had $\geq 40$ lymph nodes retrieved. On the completion of the follow-up, 34 patients deceased. The highest percentage of mortality was observed in the group of patients with $<15$ lymph nodes retrieved. The median survival probability was 83.3 months in the first group, 116.6 months in the second group and 145.6 months in the third group. Median overall survival was 35 months in group 1, 63 months in group 2 and 73.4 months in group 3 (Table III). Comparison of overall survival rates according to the defined subgroups, revealed significant differences in survival
Table I. Patient and tumor characteristics according to the number of harvested lymph nodes.

\begin{tabular}{|c|c|c|c|}
\hline & $\begin{array}{l}\text { Group } 1 \\
\text { (1-14 LNs) }\end{array}$ & $\begin{array}{c}\text { Group } 2 \\
\text { (15-39 LNs) }\end{array}$ & $\begin{array}{c}\text { Group } 3 \\
\text { (>40 LNs) }\end{array}$ \\
\hline Patients & $22(14.0 \%)$ & $79(50.3 \%)$ & $56(35.7 \%)$ \\
\hline \multicolumn{4}{|l|}{ Gender } \\
\hline Male & $20(90.9 \%)$ & $56(70.9 \%)$ & $44(78.6 \%)$ \\
\hline Female & $2(9.1 \%)$ & $23(29.1 \%)$ & $12(21.4 \%)$ \\
\hline \multicolumn{4}{|l|}{ Age (years) } \\
\hline Median & 65 & 61 & 57 \\
\hline Range & $43-77$ & $27-82$ & $39-86$ \\
\hline \multicolumn{4}{|l|}{ Localization } \\
\hline Oral cavity & $6(27.3 \%)$ & $26(32.9 \%)$ & $11(19.6 \%)$ \\
\hline Oropharynx & $7(31.8 \%)$ & $27(34.2 \%)$ & $14(25.0 \%)$ \\
\hline Hypopharynx & $2(9.1 \%)$ & $4(5.1 \%)$ & $4(7.1 \%)$ \\
\hline Larynx & $7(31.8 \%)$ & $22(27.8 \%)$ & $27(48.2 \%)$ \\
\hline \multicolumn{4}{|l|}{ Grading } \\
\hline G1 & $3(13.6 \%)$ & $12(15.2 \%)$ & $5(8.9 \%)$ \\
\hline $\mathrm{G} 2$ & $16(72.7 \%)$ & $54(68.4 \%)$ & $43(76.8 \%)$ \\
\hline G3 & $3(13.6 \%)$ & $10(12.7 \%)$ & $7(12.5 \%)$ \\
\hline G4 & 0 & $1(1.3 \%)$ & 0 \\
\hline G1-2 & 0 & $1(1.3 \%)$ & 0 \\
\hline G3-4 & 0 & 0 & $1(1.8 \%)$ \\
\hline N/A & 0 & $1(1.3 \%)$ & 0 \\
\hline \multicolumn{4}{|l|}{ T-stage } \\
\hline $\mathrm{T} 1$ & $10(45.5 \%)$ & $38(48.1 \%)$ & $19(33.9 \%)$ \\
\hline $\mathrm{T} 2$ & $8(36.4 \%)$ & $31(39.2 \%)$ & $21(37.5 \%)$ \\
\hline $\mathrm{T} 3$ & $3(13.6 \%)$ & $5(6.3 \%)$ & $12(21.4 \%)$ \\
\hline $\mathrm{T} 4$ & $1(4.5 \%)$ & $5(6.3 \%)$ & $4(7.1 \%)$ \\
\hline
\end{tabular}

LNs: Lymph nodes.

between all groups with improved survival of patients with a lymph node yield of at least 15 nodes $(p=0.001)$.

\section{Discussion}

The lymph node status has important implications for the prognosis and management of $\operatorname{HNSCC}(12,13)$. Since nodenegative tumors have less aggressive biological features and a more favorable prognosis compared to node-positive HNSCC, adequate nodal evaluation is important to initiate optimal treatment. Until now, guidelines with regard to the optimal number of sampled lymph nodes have not been established. There are several studies demonstrating that a higher lymph node yield may be correlated with improved survival in HNSCC patients receiving elective and therapeutic neck dissections $(7,8,14,15)$. This was also suggested for patients with pathological N0 neck $(7,8)$. However, the adequate lymph node yield needs to be defined. By stratifying nodal yield into quartiles, Ebrahimi et al. (7) showed that patients with oral cancer and at least 18 nodes retrieved had improved outcomes. The prognostic impact of this cut-off point was confirmed by further studies 


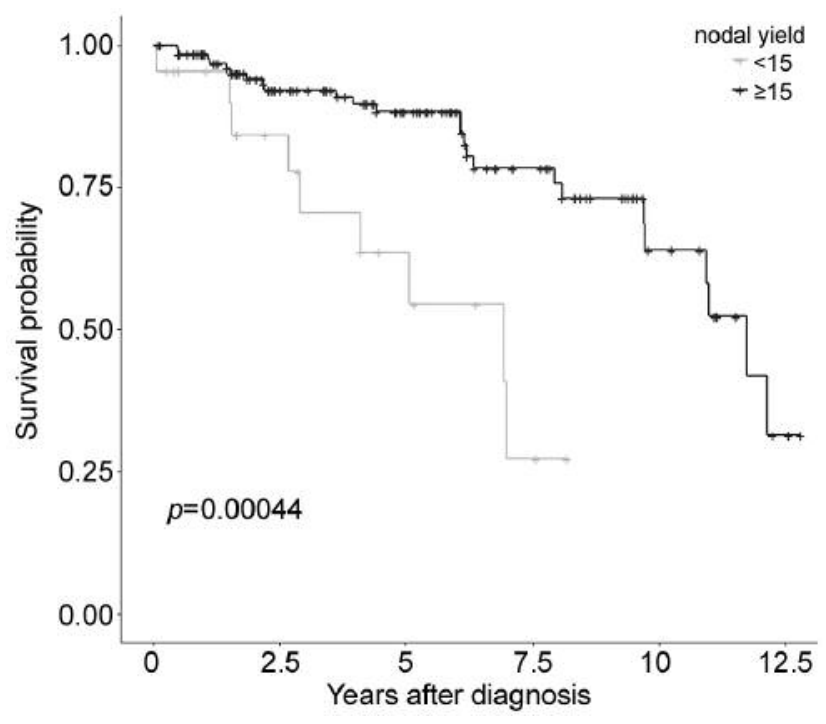

Figure 1. Kaplan-Meier survival curves for 157 patients stratified by nodal yield into two groups.

Table II. Treatment characteristics of the patients according to the number of harvested lymph nodes.

\begin{tabular}{lccc}
\hline & $\begin{array}{c}\text { Group 1 } \\
(1-14 \text { LNs })\end{array}$ & $\begin{array}{c}\text { Group 2 } \\
(15-39 \text { LNs })\end{array}$ & $\begin{array}{c}\text { Group 3 } \\
(>40 \text { LNs })\end{array}$ \\
\hline $\begin{array}{l}\text { Neck dissection } \\
\text { Ipsilateral }\end{array}$ & $18(81.8 \%)$ & $54(68.4 \%)$ & $10(17.9 \%)$ \\
$\quad$ Bilateral & $4(18.2 \%)$ & $25(3.6 \%)$ & $46(82.1 \%)$ \\
Number of & & & \\
harvested LN & & & \\
$\quad$ Median & 10.5 & 26.0 & 53.0 \\
Mean & 10.5 & 25.2 & 58.3 \\
$\quad$ Range & $6-14$ & $15-39$ & $41-124$ \\
Adjuvant treatment & $6(27.3 \%)$ & $20(25.3 \%)$ & $11(19.6 \%)$ \\
$\quad$ Radiotherapy & $1(4.5 \%)$ & $11(13.9 \%)$ & $3(5.4 \%)$ \\
Radiochemotherapy & $5(22.7 \%)$ & $9(11.4 \%)$ & $7(12.5 \%)$ \\
Chemotherapy & 0 & 0 & $1(1.8 \%)$ \\
\hline
\end{tabular}

LNs: Lymph nodes.

on oral SCC $(16,17)$. A nodal yield of more than 22 nodes was found to be a significant predictor of overall survival in patients with oral SCC and pN0 neck (8). By determining the lowest lymph node yield at which a significant survival advantage occurred, Kuo et al. (18) found that the optimal threshold of lymph node yield for $\mathrm{cN} 0$ oral SCC was 16 . The present study provided further evidence that the lymph node yield of neck dissection has an impact on prognosis in HNSCC. Patients with pathologically node-negative HNSCC with fewer than 15 lymph nodes removed had a significantly greater likelihood of overall mortality than patients with at

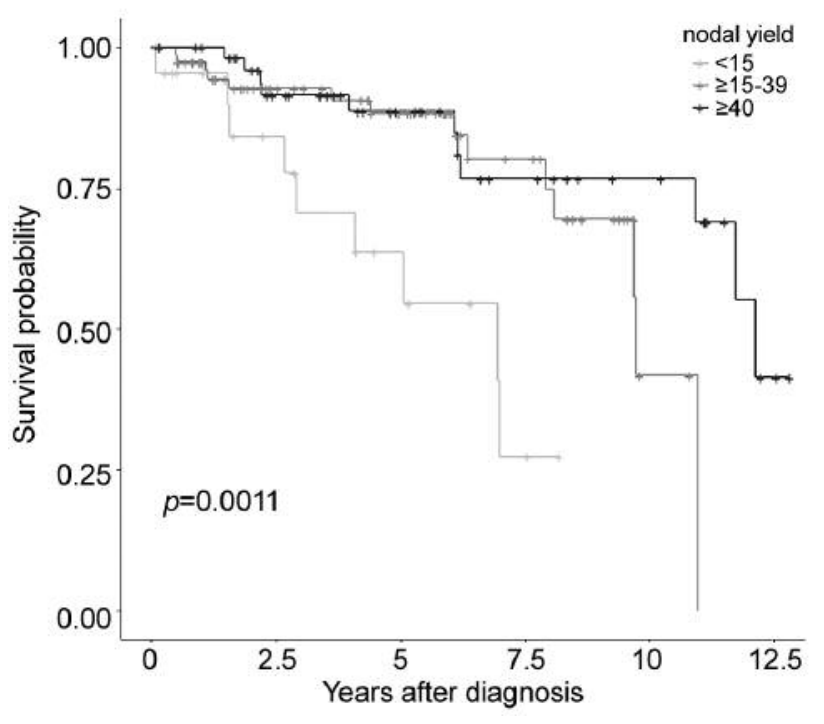

Figure 2. Kaplan-Meier survival curves for 157 patients stratified by nodal yield into three groups.

Table III. Follow-up and survival of the patient population according to the number of harvested lymph nodes.

\begin{tabular}{lccc}
\hline & $\begin{array}{c}\text { Group 1 } \\
(1-14 \text { LNs })\end{array}$ & $\begin{array}{c}\text { Group 2 } \\
(15-39 \text { LNs })\end{array}$ & $\begin{array}{c}\text { Group 3 } \\
(>40 \text { LNs })\end{array}$ \\
\hline Status at time of survey & & & \\
$\quad$ Alive & $13(59.1 \%)$ & $65(82.3 \%)$ & $45(80.4 \%)$ \\
$\quad$ Dead & $9(40.9 \%)$ & $14(17.7 \%)$ & $11(19.6 \%)$ \\
Follow-up in months & & & \\
$\quad$ Median & 34.7 & 52.5 & 58.2 \\
$\quad$ Mean & 41.9 & 52.7 & 66.0 \\
$\quad$ Range & $1.0-98.2$ & $1.3-131.8$ & $1.7-153.7$ \\
Overall survival in months & & & \\
$\quad$ Median & 35.0 & 63.0 & 73.4 \\
$\quad$ Mean & 42.5 & 61.4 & 76.1 \\
$\quad$ Range & $1.0-84$ & $5.9-132$ & $22.3-145.2$ \\
Survival probability in months & & & \\
$\quad$ Median & 83.3 & 116.6 & 145.6 \\
$\quad$ Mean & 65.3 & 106.7 & 125.4 \\
\hline
\end{tabular}

LNs: Lymph nodes.

least 15 nodes removed. The exact mechanisms behind the demonstrated survival advantage are unknown.

The extent of neck dissection and the number of lymph nodes retrieved can vary and may be impacted by numerous patient and provider factors like differences in surgical or pathologic approach, intrinsic tumor factors, and patient characteristics. An increasing number of examined lymph nodes indicates a high quality of surgical care. One possible explanation for the association between lymph node yield and survival is that a more extensive neck dissection may result in eradication of micro-metastatic disease and 
therefore leads to less understaging, which in turn leads to better treatment and survival. Another explanation is that the lymph node yield is a surrogate for patient and provider factors that more directly influence survival. Furthermore, studies on colorectal cancer found that a stronger lymphocyte response against tumor cells may be related to the number of removed lymph nodes $(19,20)$.

The impact of nodal yield on outcome may be altered by adjuvant radio(chemo)therapy which reduces the higher risk of regional failure associated with low nodal yield. However, in our study population patients with low nodal yield (group 1) and poorest survival had the highest rates of adjuvant radio(chemo)therapy.

Given its retrospective nature, the study has several limitations. The first and most obvious is that it consists of a relatively small number of patients with different tumor locations. Inherent to the design we have to deal with missing data which could have impacted our results. Survival data were limited to only overall survival; therefore, disease-specific mortality or disease-free survival could not be assessed.

\section{Conclusion}

In the present study the nodal yield was associated with overall survival in patients with pNO HNSCC, which is in line with several previous studies. Further, robust prospective clinical studies are needed to define the adequate nodal yield.

\section{References}

1 D'Cruz AK, Vaish R, Kapre N, Dandekar M, Gupta S, Hawaldar R, Agarwal JP, Pantvaidya G, Chaukar D, Deshmukh A, Kane S, Arya S, Ghosh-Laskar S, Chaturvedi P, Pai P, Nair S, Nair D and Badwe R; Head and neck disease management group: elective versus therapeutic neck dissection in node-negative oral cancer. N Engl J Med 373: 521-529, 2015.

2 van Erning FN, Crolla RM, Rutten HJ, Beerepoot LV, van Krieken JH and Lemmens VE: No change in lymph node positivity rate despite increased lymph node yield and improved survival in colon cancer. Eur J Cancer 50: 3221-3229, 2014.

3 Weir L, Speers C, D'yachkova Y and Olivotto IA: Prognostic significance of the number of axillary lymph nodes removed in patients with node-negative breast cancer. J Clin Oncol 20: 1793-1799, 2002.

4 Brenkman HJF, Goense L, Brosens LA, Haj Mohammad N, Vleggaar FP, Ruurda JP and van Hillegersberg R: A high lymph node yield is associated with prolonged survival in elderly patients undergoing curative gastrectomy for cancer: A Dutch populationbased cohort study. Ann Surg Oncol 24: 2213-2223, 2017.

5 Altorki NK, Zhou XK, Stiles B, Port JL, Paul S, Lee PC and Mazumdar M: Total number of resected lymph nodes predicts survival in esophageal cancer. Ann Surg 248: 221-226, 2008.

6 Li R, Petros FG, Davis JW: Extended pelvic lymph node dissection in bladder cancer. J Endourol 32(S1): S49-S54, 2018.

7 Ebrahimi A, Clark JR, Amit M, Yen TC, Liao CT, Kowalski LP, Kreppel M, Cernea CR, Bachar G, Villaret AB, Fliss D, Fridman
E, Robbins KT, Shah JP, Patel SG and Gil Z: Minimum nodal yield in oral squamous cell carcinoma. Defining the standard of care in a multicenter international pooled validation study. Ann Surg Oncol 21: 3049-3055, 2014.

8 Lemieux A, Kedarisetty S, Raju S, Orosco R and Coffey C: Lymph node yield as a predictor of survival in pathologically node negative oral cavity carcinoma. Otolaryngol Head Neck Surg 154: 465-472, 2016.

9 Contal C and O'Quigley J: An application of changepoint methods in studying the effect of age on survival in breast cancer. Comput Stat Data Anal 30: 253-270, 1999.

10 Mandrekar JN, Mandrekar SJ and Cha SS: Cutpoint determination methods in survival analysis using $\mathrm{SAS}^{\circledR}$. Proceedings of the 28th SAS Users Group International Conference (SUGI 28) (Paper 261-28), 2003.

11 Harrington DP and Fleming TR: A class of rank test procedures for censored survival data. Biometrika 69: 553-566, 1982.

12 Noble AR, Greskovich JF, Han J, Reddy CA, Nwizu TI, Khan MF, Scharpf J, Adelstein DJ, Burkey BB and Koyfman SA: Risk factors associated with disease recurrence in patients with stage III/IV squamous cell carcinoma of the oral cavity treated with surgery and postoperative radiotherapy. Anticancer Res 36: 785-792, 2016.

13 Wiegand S, Esters J, Müller HH, Jäcker T, Papaspyrou G, Roessler M, Werner JA and Sesterhenn AM: Relevance of oropharyngeal cancer lymph node metastases in the submandibular triangle and the posterior triangle apex. Anticancer Res 29: 4785-4790, 2009.

14 Shrime MG, Ma C, Gullane PJ, Gilbert RW, Irish JC, Brown DH and Goldstein DP: Impact of nodal ratio on survival in squamous cell carcinoma of the oral cavity. Head Neck 31: 1129-1136, 2009.

15 Süslü N, Hoşal AS and Sözeri B: Prognostic value of metastatic lymph node ratio in node-positive head and neck carcinomas. Am J Otolaryngol 31: 315-319, 2010.

16 Divi V, Harris J, Harari PM, Cooper JS, McHugh J, Bell D, Sturgis EM, Cmelak AJ, Suntharalingam M, Raben D, Kim H, Spencer SA, Laramore GE, Trotti A, Foote RL, Schultz C, Thorstad WL, Zhang QE, Le QT and Holsinger FC: Establishing quality indicators for neck dissection: correlating the number of lymph nodes with oncologic outcomes (NRG Oncology RTOG 9501 and RTOG 0234). Cancer 122: 3464-3471, 2016.

17 Divi V, Chen MM, Nussenbaum B, Rhoads KF, Sirjani DB, Holsinger FC, Shah JL and Hara W: Lymph node count from neck dissection predicts mortality in head and neck cancer. $\mathrm{J}$ Clin Oncol 34: 3892-3897, 2016.

18 Kuo P, Mehra S, Sosa JA, Roman SA, Husain ZA, Burtness BA, Tate JP, Yarbrough WG and Judson BL: Proposing prognostic thresholds for lymph node yield in clinically lymph nodenegative and lymph node-positive cancers of the oral cavity. Cancer 122: 3624-3631, 2016.

19 Pagès F, Galon J and Fridman WH: The essential role of the in situ immune reaction in human colorectal cancer. J Leukoc Biol 84: 981-987, 2008.

20 Morris M, Platell C and Lacopetta B: Tumor-infiltrating lymphocytes and perforation in colon cancer predict positive response to 5-fluorouracil chemotherapy. Clin Cancer Res 14: 1413-1417, 2008.

Received July 22, 2018

Revised July 30, 2018

Accepted August 1, 2018 